\title{
The Effect of Plate Rotation on the Stiffness of Femoral LISS: A Mechanical Study
}

\author{
Afshin Khalafi, MD, Shane Curtiss, RA, Scott Hazelwood, PhD, and Philip Wolinsky, MD
}

\begin{abstract}
Objective: Malposition of the femoral Less Invasive Stabilization System (LISS) plate may alter its biomechanical behavior. This study compares the mechanical stability of "correctly" affixed LISS plates matching the slope of the lateral femoral condyle to "incorrectly" placed LISS plates fixed in external rotation relative to the distal femur.
\end{abstract}

Methods: A fracture gap model was created to simulate a comminuted supracondylar femur fracture (AO/OTA33-A3). Fixation was achieved using two different plate positions: the LISS plate was either placed "correctly" by internally rotating the plate to match the slope of the lateral femoral condyle, or "incorrectly" by externally rotating the plate relative to the distal femur. Following fixation, the constructs were loaded in axial, torsional, and cyclical axial modes in a material testing machine.

Main Outcome Measurement: Stiffness in axial and torsional loading; total deformation and irreversible (plastic) deformation in cyclical axial loading.

Results: The mean axial stiffness for the correctly placed LISS constructs was $21.5 \%$ greater than the externally rotated LISS constructs $(62.7 \mathrm{~N} / \mathrm{mm}$ vs. $49.3 \mathrm{~N} / \mathrm{mm} ; P=0.0007)$. No significant difference was found in torsional stiffness between the two groups. Cyclical axial loading caused significantly less $(P<0.0001)$ plastic deformation in the correct group $(0.6 \mathrm{~mm})$ compared with externally rotated group $(1.3 \mathrm{~mm})$. All the constructs in the incorrect group failed, where failure was defined as a complete closure of the medial fracture gap, prior to completion of the test cycles.

Conclusion: Correct positioning of the LISS plate for fixation of distal femur fractures results in improved mechanical stability as reflected by an increased stiffness in axial loading and decreased plastic deformation at the bone-screw interface.

Key Words: Less Invasive Stabilization System, mechanical testing, distal femur fractures, stiffness, modes of failure

\section{INTRODUCTION}

Recent trends in fracture care with emphasis on "biologic fixation" have lead to the development of the Less Invasive Stabilization System (LISS; Synthes, Paoli, PA), ${ }^{1-5}$ which was developed to minimize fracture site soft-tissue dissection while maximizing fixation and stability of fractures of the distal femur. ${ }^{6-8}$

The LISS behaves as an internal splint, with each locked screw acting as a fixed-angle device. Because screw toggling is minimized in the screw-plate interface, the LISS construct usually fails in one of two modes: (1) all diaphyseal screws slice through the cortex in a longitudinal manner or (2) all pull out of the bone. ${ }^{9}$ Schutz et al, in a study of 107 distal femur fractures stabilized using the LISS plate, found 4 cases of implant loosening and 2 cases of implant breakage. The unicortical diaphyseal screws were the ones that always loosened. ${ }^{10}$ Four causes of failure were suggested: an implant that was too short, failure of the screws to lock into the implant, ventral positioning of the plate on the femoral shaft resulting in insufficient fixation of the diaphyseal unicortical screws, and immediate full weight-bearing in a psychiatric patient. ${ }^{10}$ Schandelmaier et al noted that 4 of 54 patients with distal femur fractures had proximal screw pullout. This was attributed to malposition/rotation of the LISS plate, leading to a tangential placement of the unicortical diaphyseal screws. ${ }^{11}$ Loosening of the screws placed in the femoral condyles has not been reported for the LISS plate.

This raised the question whether the LISS system has two modes of failure dependent on how the diaphyseal screws are placed. ${ }^{12}$ If the shaft screws are placed tangentially, as a result of a malposition of the plate, then only a portion of the screw threads actually obtain purchase in the femoral cortex and the system fails by these screws "pulling off" the femoral shaft. However, proper placement of the plate leads to more screw threads gaining purchase in the femoral cortex of the diaphysis, and more secure fixation may lead to failure of the plate rather than "pull out" of the proximal screws. $^{12}$

To our knowledge, no previous study has compared the mechanical stability of femoral LISS plates placed "incorrectly" in external rotation relative to the lateral femoral condyle, resulting in tangential placement of diaphyseal screws, to plates affixed "correctly" matching the slope of the distal femur, with central placement of proximal shaft screws. Thus, the purpose of our study was to measure whether correct rotational positioning of the LISS plate would show increased stiffness compared to incorrect positioning. 


\section{MATERIALS AND METHODS}

\section{Fracture Model}

All the osteotomies were performed after the application of the LISS plate by an experienced fellowship-trained orthopaedic trauma surgeon. An AO/OTA33-A3 fracture model was created in all specimens. A 1-cm gap was created $6 \mathrm{~cm}$ proximal to the intercondylar notch to mimic an unstable fracture pattern with loss of stability of the medial and lateral columns of the distal femur. An additional 3-cm diagonal cut was made in the proximal medial cortex to prevent bone-to-bone contact during testing, as described by Zlowodzki et $\mathrm{al}^{13}$ (Fig. 1).

\section{Construct Design}

A 9-hole femoral LISS template was used to ensure consistent plate placement in each group. Two construct groups were created using $3^{\text {rd }}$-generation femoral synthetic composite bones (Sawbones 3306, Pacific Research Laboratories, Inc., Vashon, WA). The use of Sawbones for mechanical testing is well established and eliminates the variation in stiffness found in cadaver bones. ${ }^{14,15}$ Each group contained 9 specimens:

\section{Group 1, "Correct” or Internally Rotated Constructs}

The Sawbones were instrumented using a 9-hole LISS plate. The plate was internally rotated by 10 degrees to match the slope of the lateral femoral condyle. Proximal fixation was achieved with 26- $\mathrm{mm}$ long unicortical screws $(5.0 \mathrm{~mm}$ in diameter) placed in screw holes $9,7,5$, and 3 (hole 9 was the most proximal screw hole). Five unicortical screws were

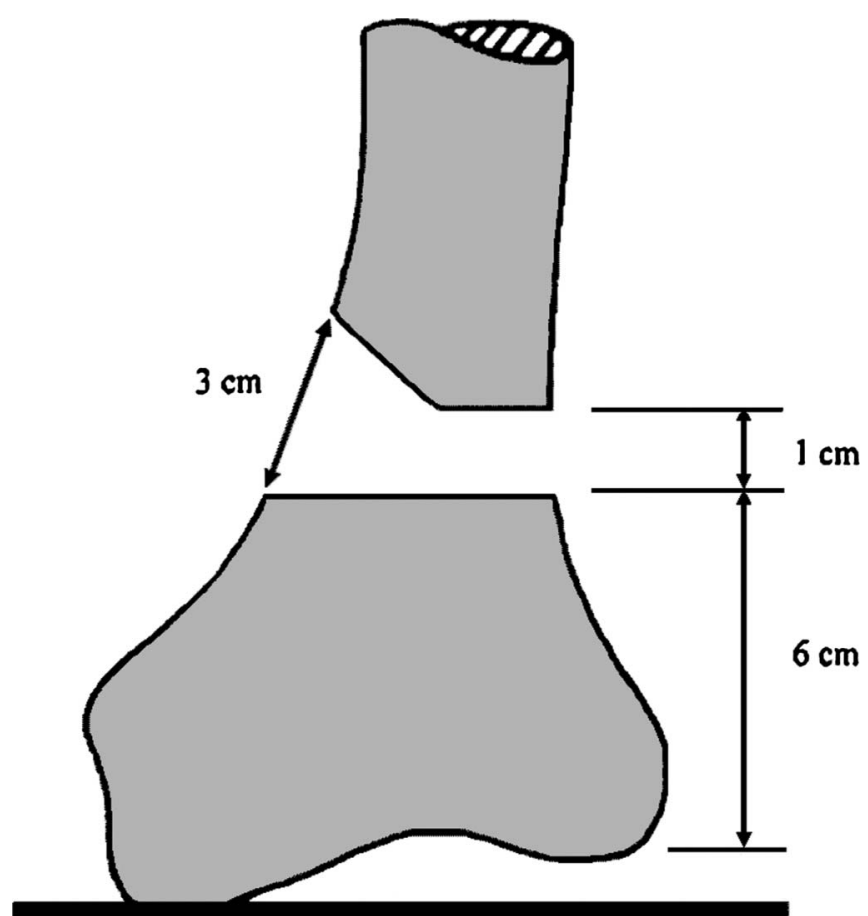

FIGURE 1. A Sawbone model simulating an AO/OTA33-A3 fracture was used. A $1-\mathrm{cm}$ fracture gap was created $6 \mathrm{~cm}$ proximal to the intercondylar notch. Additionally, a $3-\mathrm{cm}$ diagonal cut was made in the proximal medial cortex to prevent bone-to-bone contact during mechanical testing. placed in screw holes D, E, F, C, and G to obtain distal fixation. Distal screw length was based on the width of the femoral condyles. With the internal rotation of the LISS plates in this group the proximal screw holes ended up centered on the femoral shaft, resulting in a "correct" nontangential placement of the diaphyseal screws (Fig. 2A).

\section{Group 2, "Incorrect" or Externally Rotated Constructs}

The Sawbones were instrumented using a 9-hole LISS plate. Proximal and distal fixation was achieved using the method described for Group 1, except the plates were not internally rotated to match the slope of the lateral femoral condyles in this group. Instead, the plates were externally rotated relative to the slope of the distal femur by 15 degrees, causing a ventral shift in the diaphyseal portion of the LISS plate. This resulted in tangential placement of the diaphyseal screws in the anterior femoral cortex with less screw threads engaged in the cortex (Fig. 2B).

\section{Mechanical Testing}

After instrumentation and osteotomy, the proximal half and distal end of each femur were held in a custom-built polymethylmethacrylate (PMMA) mold. The position of each femur in the mold was such that the line of action for the load went through the center of the femoral head and the intercondylar notch, simulating the mechanical axis of the femur. This model was used to load the constructs in axial and cyclic loading and was based on a loading apparatus
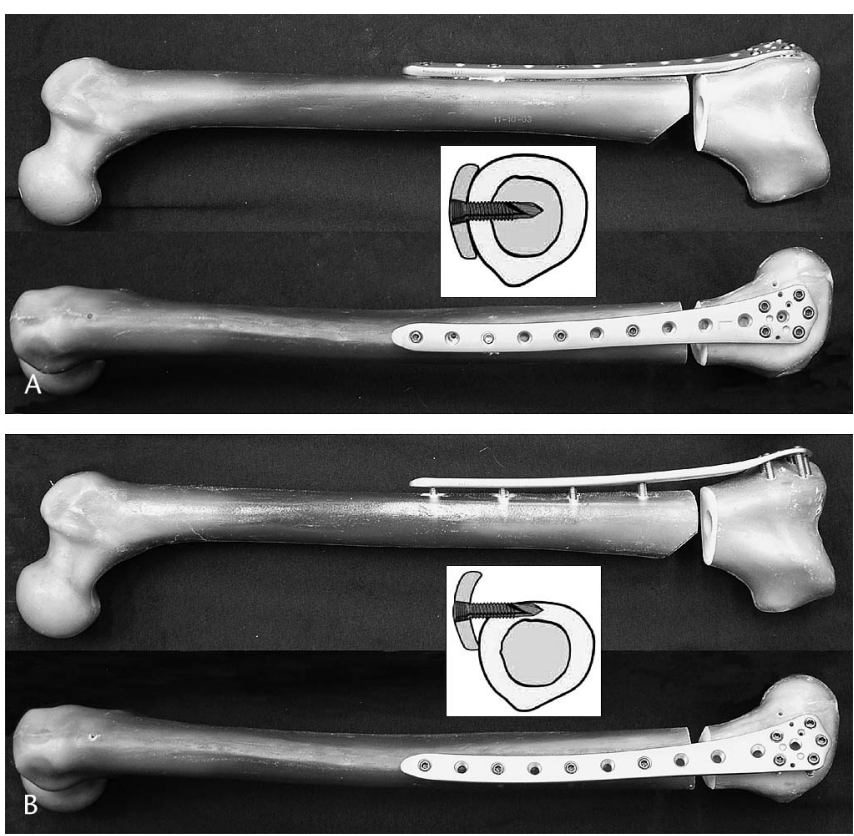

FIGURE 2. A, "Correct" construct group: LISS plates were internally rotated to match the slope of the lateral femoral condyle, resulting in a nontangential (centered) placement of the diaphyseal screws. B, "Incorrect" construct group: LISS plates were externally rotated by 15 degrees relative to the femoral shaft, causing a slight ventral shift in the proximal LISS plate with subsequent tangential placement of the diaphyseal screws in the anterior femoral cortex. 
previously described by Cordey et al. ${ }^{21}$ Our version was simplified by potting the proximal part of the femur. Our main objective was to physiologically simulate the line of force in the frontal and sagittal planes at the distal femur.

The model was then placed on the loading platform of a materials testing machine (Instron $5800 \mathrm{R}$, Canton, MA) for mechanical testing (Fig. 3). The specimens were supported by a ball bearing in the testing machine to avoid uncontrolled torque or bending. ${ }^{21}$ For torsional testing, the specimens were proximally held in a custom mold and distally secured in a chuck, with the femoral axis in line with the axis of rotation. The apparatus was then connected to the actuator of the Instron (Fig. 4).

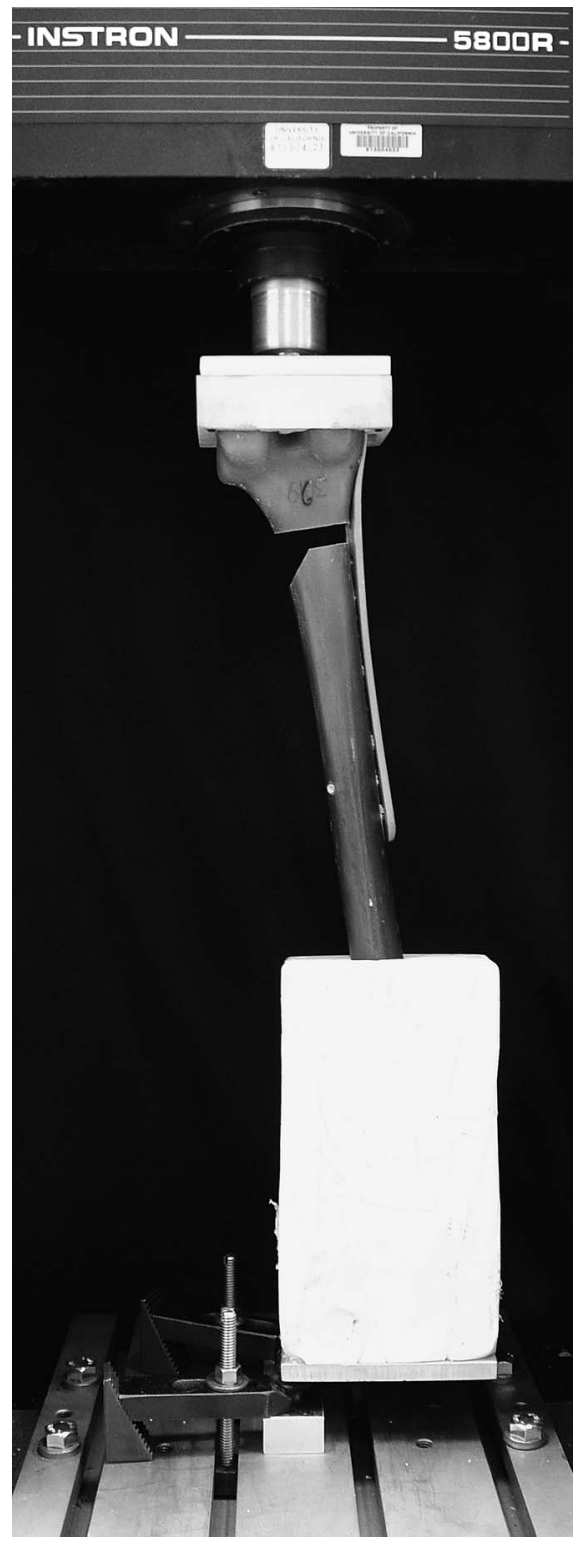

FIGURE 3. For axial and cyclical axial testing, the proximal half and distal end of each femur were held in a PMMA mold. The model was proximally and distally supported by a ball bearing in the materials testing machine to avoid uncontrolled torque or bending.

\section{TESTING PROTOCOL}

\section{Axial Loading}

The constructs were loaded in compression at a loading rate of $10 \mathrm{~mm} / \mathrm{min}$. After stabilizing the construct with a preload of $100 \mathrm{~N}$, axial loading was performed in a displacement control mode. Testing was stopped when either $500 \mathrm{~N}$ was reached, or failure occurred as defined by a complete medial fracture gap closure or visual loss of fixation.

\section{Torsional Loading}

The specimens were preloaded to $5 \mathrm{Nm}$ and each construct was torqued to a maximum of $20 \mathrm{Nm}$ at a rate of about 20 degrees/min. Torsion resulted in internal rotation of the femur relative to the LISS plate. Testing was stopped when either visual loss of fixation occurred or any of the shaft screws pulled out.

\section{Cyclical Axial Loading}

This loading protocol was previously described for the mechanical evaluation of distal femur fractures. ${ }^{13,16}$ It consisted of increments of 10 cycles starting with $300 \mathrm{~N}$. The load for each successive increment was increased by $100 \mathrm{~N}$, to a maximum load of $1000 \mathrm{~N}$, with 10 seconds of rest between each increment. The preload and baseline load after each cycle was $100 \mathrm{~N}$. Testing was conducted in a displacement control mode at $0.75 \mathrm{~mm} / \mathrm{sec}$ and was performed until visual loss of fixation occurred or the medial fracture gap completely closed.

This method of cyclical testing was used to compare the influence of correct versus incorrect plate placement on screw fixation in the femoral diaphysis and to assess their contribution to reversible and irreversible (plastic) deformation.

\section{Data Recording and Statistical Analysis}

For axial and torsional testing, a load-displacement curve was plotted for each construct (Microsoft Excel, Seattle,

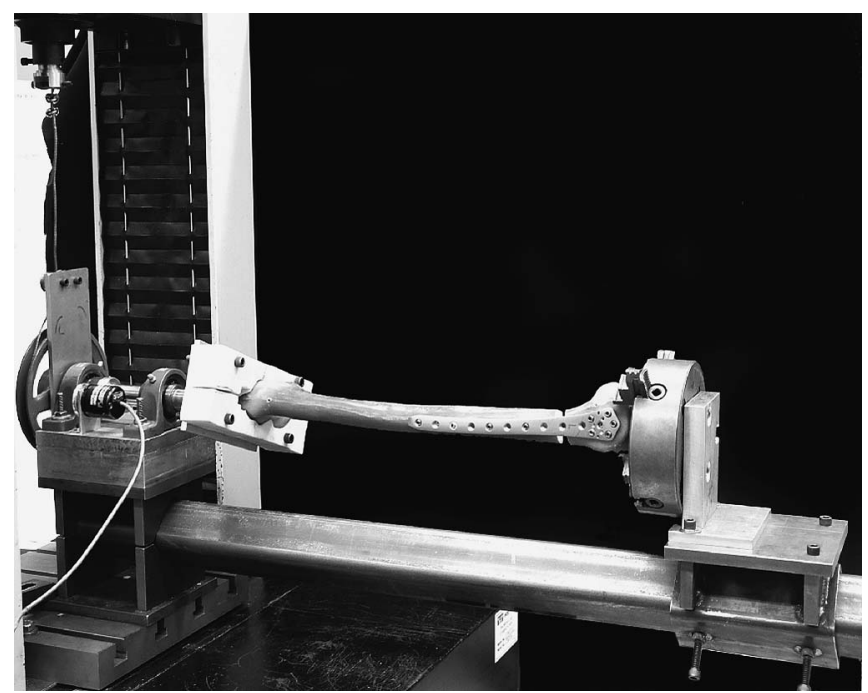

FIGURE 4. For torsional testing, the proximal femur was held in a PMMA mold and distally the condyles were secured in a lathe chuck. Torsion occurred through a cable connected to the actuator of the Instron, resulting in internal rotation of the femur relative to the LISS plate. 
WA) and the stiffness was calculated as the slope of the initial region of the curve. Reversible and irreversible deformation in cyclical axial loading is shown in a typical time-displacement curve of the LISS construct (Fig. 5). Plastic deformation was calculated by subtracting the amount of displacement present at the start of the first cycle $(300 \mathrm{~N})$ from displacement present after the final cycle. Total deformation was recorded after the last testing cycle. ${ }^{16}$

A one-way analysis of variance (ANOVA) was performed using StatView (SAS Institute Inc., Cary, NC) to determine statistically significant differences in axial and torsional stiffness and plastic deformation between each group. The level of significance was defined as $P \leq 0.05$.

\section{RESULTS}

\section{Axial/Torsional Loading}

No visual loss of fixation or complete closure of the medial fracture gap occurred in either the axial or the torsional loading groups. The mean axial stiffness for the correctly placed LISS plate constructs was $21.5 \%$ higher than mean stiffness for the externally rotated plate constructs (Table 1). Although this represented a statistically significant difference in axial loading $(P=0.0007)$, a difference of only $1.8 \%$ in stiffness was found between the two groups in torsional loading $(P=0.1893)$. None of the shaft screws pulled out of the anterior cortex.

\section{Cyclical Axial Loading}

After each set of loading cycles, the amount of irreversible/plastic deformation increased significantly more in the incorrect plate group than in the correct plate group

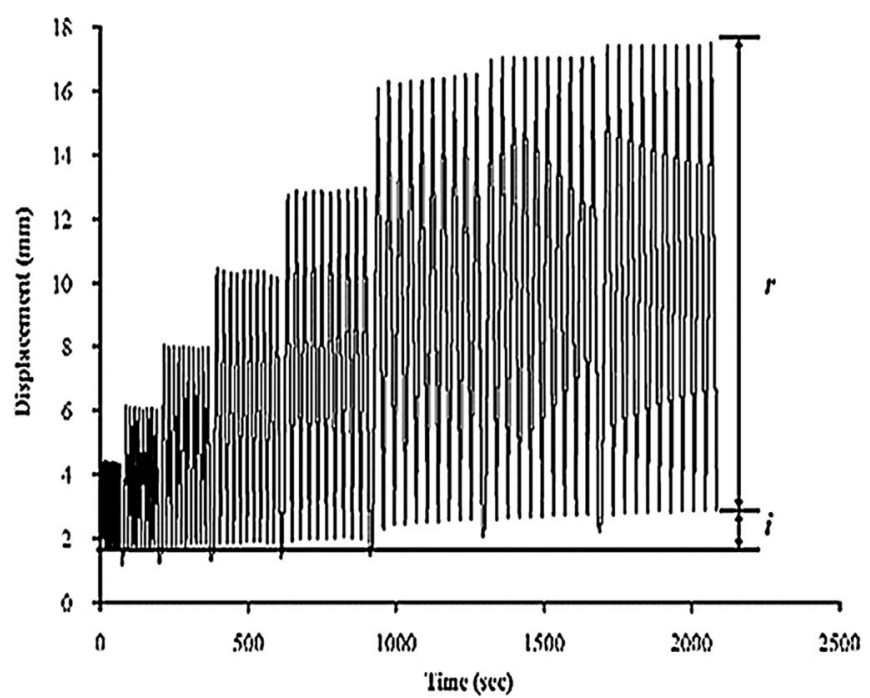

FIGURE 5. A typical time versus displacement curve for cyclical axial testing. The maximal load applied was increased in 8 increments of 10 cycles, starting with $300 \mathrm{~N}$. Each successive increment was increased by $100 \mathrm{~N}$, to a maximum load of $1000 \mathrm{~N}$, followed by 10 seconds of rest. The preload and baseline load after each increment was $100 \mathrm{~N}$. Letters ( $I$ ) and $(r)$ represent irreversible (plastic) and reversible deformation, respectively.
TABLE 1. Stiffness, Total and Plastic Deformations for Femoral LISS Plate Constructs as a Function of Plate Position

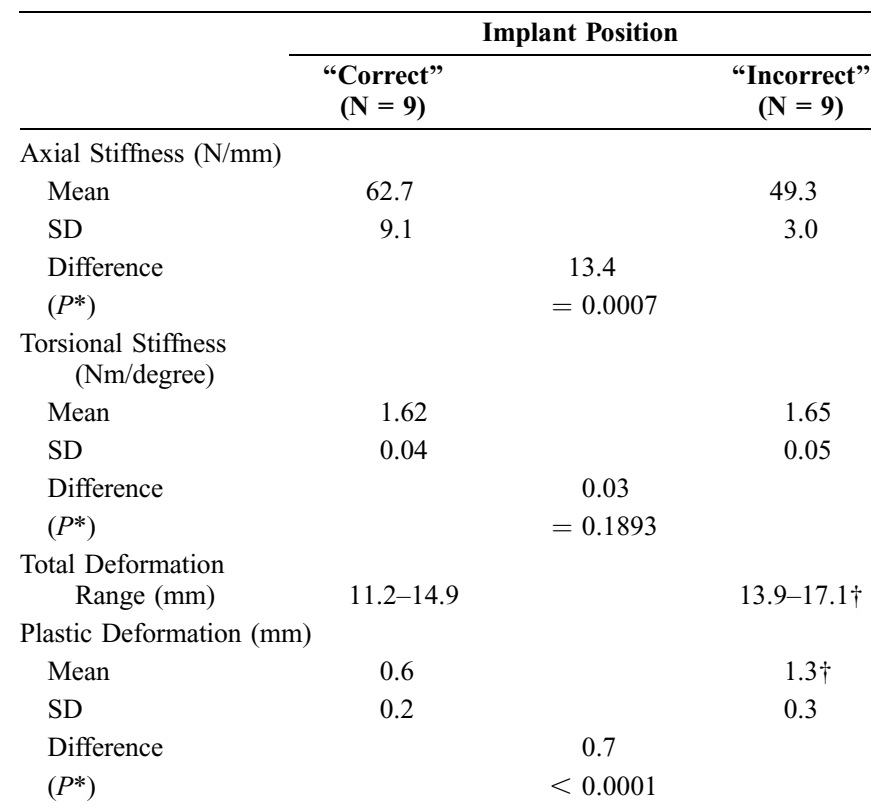

* One way analysis of variance (ANOVA)

†Values represent only test cycles of up to $900 \mathrm{~N}$. Testing was stopped at the end of the $800 \mathrm{~N}$ cycle increment because medial fracture gap completely closed in all the constructs.

(Table 1). No visual loss of fixation occurred in either group. However, failure, defined as complete closure of the medial osteotomy gap, occurred in all incorrect constructs at the end of the 800-N cycle increment. Complete closure of the fracture gap did not occur for any of the correct constructs throughout the duration of the test.

The mean plastic deformation was calculated by subtracting the amount of displacement present at the beginning of the $300 \mathrm{~N}$ cycles, from the displacement present at the end of the $1000 \mathrm{~N}$ cycles for the correct group, and from displacement present at the point of medial gap closure (end of the $800 \mathrm{~N}$ cycle) for the incorrect group, respectively. The incorrect constructs had $55 \%$ more irreversible deformation than the correct constructs $(P<0.0001)$.

\section{DISCUSSION}

Biomechanical studies often correlate the stability of a construct with its stiffness. ${ }^{17-20}$ A stiffer construct is thought to be more stable because it allows for less motion at the fracture site. We found that the correct group was stiffer in axial loading but not in torsional loading. Our findings in axial loading were expected because plates placed centrally on the mid lateral aspect of the femur ensure proper positioning and better purchase of the proximal diaphyseal screws in the femoral cortex. Hence, in axial loading retention forces are more evenly distributed among the shaft screws, resulting in a more stable fixation. ${ }^{11}$

In the externally rotated group, we expected the diaphyseal screws to cut out the anterior femoral cortex, given the tangential placement of these screws. Because less 
cortical bone is available to resist torsional moments acting on the diaphyseal screws, we anticipated significant differences between the two constructs; pull out of these screws under physiologic loading has been reported in the literature as a potential mode of failure for the femoral LISS plate. ${ }^{9,10,12}$ However, none of the screws in our study pulled out. This may be explained by the fact that under physiologic loading conditions, pure torsional moments acting on the distal femur are not likely, a torque in excess of $20 \mathrm{Nm}$ might be necessary to cause an anterior pull out of the shaft screws, or both.

In a study of distal femur fractures, Marti et $\mathrm{al}^{16}$ compared the mechanical stability of the femoral LISS plate with conventional plating systems that used bicortical nonlocking screws, such as Dynamic Condylar Screw (DCS) and Condylar Buttress Plate (CBP). They concluded that the LISS construct was less stiff (more elastic) and underwent less irreversible or plastic deformation (subsidence). Plastic deformation was attributed to two main factors: (1) toggling between screws and the plate, and (2) bone destruction in the anchoring region caused by excessive stress in the bone-screw interface, leading to irreversible sinking of the screws into the supporting bone. Because LISS is a locked screw-plate construct, plastic deformation can be explained by the contribution of bone destruction.

According to our data, the amount of plastic deformation at the screw-bone interface is greater in an externally rotated incorrectly placed plate model. This is likely secondary to an uneven stress distribution among the tangentially placed screws that have less purchase in the cortex when compared with correctly placed screws. The magnitude of irreversible deformation among the incorrect LISS constructs is likely an underestimation of the actual value because testing was stopped at the end of the $800 \mathrm{~N}$ cycles secondary to a complete closure of the medial fracture gap.

An inherent limitation of mechanical studies is their inability to accurately reproduce both the internal and external loading environment of the distal femur. We chose our cyclical axial loading protocol because it has been used by other investigators to simulate physiologic loading conditions in the distal femur. ${ }^{13,16}$ Although this model did not take into account the actual muscle forces acting in the distal femur, we feel that it was appropriate for comparing the relative stability and stiffness of the two construct groups.

In light of the previously mentioned observations and previous clinical studies, ${ }^{3-5,8-10,12}$ we feel that our hypothesis is supported; correct placement of the LISS plate creates a more rigid and stable fixation, as reflected by a significant increase in axial stiffness and a significant decrease in plastic deformation at the bone-screw interface. When using a LISS plate to stabilize a distal femur fracture, attention must be paid to proper positioning of the plate; it must be internally rotated to match the slope of the lateral femoral condyle, ensuring central placement of the proximal diaphyseal screws.

\section{ACKNOWLEDGMENT}

The authors would like to thank Synthes USA for providing the implants and synthetic composite bone specimens utilized in this study. We also like to thank Dr. David
Fyhrie, David Linn Chair in the University of California, Davis, Department of Orthopaedics, for his assistance in designing the mechanical testing model.

\section{REFERENCES}

1. Frigg R, Appenzeller A, Christensen R, et al. The development of the distal femur Less Invasive Stabilization System (LISS). Injury. 2001; 32(Suppl 3):SC24-31.

2. Krettek C, Muller M, Miclau T. Evolution of minimally invasive plate osteosynthesis (MIPO) in the femur. Injury. 2001;32(Suppl 3):SC14-23.

3. Weight M, Collinge C. Early results of the less invasive stabilization system for mechanically unstable fractures of the distal femur (AO/OTA types A2, A3, C2, and C3). J Orthop Trauma. 2004;18:503-508.

4. Syed AA, Agarwal M, Giannoudis PV, et al. Distal femoral fractures: long-term outcome following stabilisation with the LISS. Injury. 2004;35: 599-607.

5. Schutz M, Muller M, Regazzoni $P$, et al. Use of the Less Invasive Stabilization System (LISS) in patients with distal femoral (AO33) fractures: a prospective multicenter study. Arch Orthop Trauma Surg. 2005; 125:102-108

6. Kregor PJ, Hughes JL, Cole PA. Fixation of distal femoral fractures above total knee arthroplasty utilizing the Less Invasive Stabilization System (L.I.S.S.). Injury. 2001;32(Suppl 3):SC64-75.

7. Schutz M, Muller M, Kaab M, et al. Less invasive stabilization system (LISS) in the treatment of distal femoral fractures. Acta Chir Orthop Traumatol Cech. 2003;70:74-82

8. Kregor PJ, Stannard JA, Zlowodzki M, et al. Treatment of distal femur fractures using the less invasive stabilization system: surgical experience and early clinical results in 103 fractures. J Orthop Trauma. 2004;18: $509-520$.

9. Kregor PJ, Stannard JA, Zlowodzki M, et al. Distal femoral fracture fixation utilizing the Less Invasive Stabilization System (L.I.S.S.): the technique and early results. Injury. 2001;32(Suppl 3):SC32-47.

10. Schutz M, Muller M, Krettek C, et al. Minimally invasive fracture stabilization of distal femoral fractures with the LISS: a prospective multicenter study. Results of a clinical study with special emphasis on difficult cases. Injury. 2001;32(Suppl 3):SC48-54.

11. Schandelmaier P, Partenheimer A, Koenemann B, et al. Distal femoral fractures and LISS stabilization. Injury. 2001;32(Suppl 3):SC55-63.

12. Button G, Wolinsky P, Hak D. Failure of less invasive stabilization system plates in the distal femur: a report of four cases. J Orthop Trauma. 2004; $18: 565-570$.

13. Zlowodzki M, Williamson S, Cole PA, et al. Biomechanical evaluation of the less invasive stabilization system, angled blade plate, and retrograde intramedullary nail for the internal fixation of distal femur fractures. J Orthop Trauma. 2004;18:494-502.

14. Cristofolini L, Viceconti M, Cappello A, et al. Mechanical validation of whole bone composite femur models. J Biomech. 1996;29:525-535.

15. Heiner AD, Brown TD. Structural properties of a new design of composite replicate femurs and tibias. $J$ Biomech. 2001;34:773-781.

16. Marti A, Fankhauser C, Frenk A, et al. Biomechanical evaluation of the less invasive stabilization system for the internal fixation of distal femur fractures. J Orthop Trauma. 2001;15:482-487.

17. Jaakkola JI, Lundy DW, Moore T, et al. Supracondylar femur fracture fixation: mechanical comparison of the 95 degrees condylar side plate and screw versus 95 degrees angled blade plate. Acta Orthop Scand. 2002;73: $72-76$.

18. Hora N, Markel DC, Haynes A, et al. Biomechanical analysis of supracondylar femoral fractures fixed with modern retrograde intramedullary nails. J Orthop Trauma. 1999;13:539-544.

19. Koval KJ, Kummer FJ, Bharam S, et al. Distal femoral fixation: a laboratory comparison of the 95 degrees plate, antegrade and retrograde inserted reamed intramedullary nails. J Orthop Trauma. 1996;10:378-382.

20. Koval KJ, Hoehl JJ, Kummer FJ, et al. Distal femoral fixation: a biomechanical comparison of the standard condylar buttress plate, a locked buttress plate, and the 95-degree blade plate. J Orthop Trauma. 1997; 11:521-524.

21. Cordey J, Borgeaud M, Frankle M, et al. Loading model for the human femur taking the tension band effect of the ilio-tibial tract into account. Injury. 1999;30(Suppl 1):A26-30. 